\title{
Loss Analysis of Thermal Reservoirs for Electrical Energy Storage Schemes
}

\author{
Alexander J. White \\ Hopkinson Laboratory, \\ Cambridge University Engineering Department, \\ Trumpington Street, Cambridge, CB2 1PZ, UK. \\ Email:ajw36@cam.ac.uk
}

\begin{abstract}
The paper presents an analysis of thermodynamic losses in thermal reservoirs due to irreversible heat transfer and frictional effects. The focus is upon applications to large-scale electricity storage for which it is the loss in availability (or exergy) that is most relevant. Accordingly, results are presented as loss coefficients which are defined as the fractional loss of the entering availability. Only losses stemming from irreversibility are considered - heat losses to the surroundings are not included in the analysis. A number of simplifying assumptions have been adopted, but the results nonetheless clearly demonstrate the dependence of the losses on operating temperatures, reservoir geometry and mode of operation, and point the way towards methods of optimisation. Estimates for a typical installation suggest that the losses are not insignificant, particularly for one-off charge and discharge (i.e., for long term storage), but are not so large as to rule out the use of thermal reservoirs for electricity storage schemes.
\end{abstract}

Key words: Regenerators, Thermal Energy Storage, Electrical Energy Storage, Thermodynamic Loss. 


\section{INTRODUCTION}

The increasing use of renewable energy technologies for electricity generation, many of which have an unpredictably intermittent nature, will inevitably lead to a greater interest in large-scale electrical energy storage schemes. In particular, the expanding fraction of electricity produced by wind turbines will require either backup or storage capacity to cover extended periods of wind lull. The scale of this problem within the UK is discussed by Mackay [1] who points out that country-wide wind lulls of several days duration are common. Future requirements for storage will clearly depend on the future mix of generating technologies but, based on recent trends and the current emphasis on $\mathrm{CO} 2$ reduction, it is likely that several hundreds of GWh storage capacity will be required in the UK within the next few decades. (For comparison, the capacity of Britain's pumped hydro storage schemes currently totals some 30 GWh, with a power capacity of just under $3 \mathrm{GW}$.)

For large-scale storage (i.e., hundreds of MWh and upwards) the main technologies currently employed are pumped hydro storage (PHS), and compressed air energy storage (CAES). Of these, PHS is the most mature, and benefits from fast response and high round-trip efficiencies - typically 70 to $80 \%$ but suffers from high capital cost and from obvious geographical constraints. Similar limitations apply to CAES since large, robust caverns are required for storage of the air at pressures of up to 100 bar. Fuel cells, flywheels and other new storage technologies have not yet reached a stage where they can compete in terms of cost, capacity and power output with either PHS or CAES.

Thermal energy storage is employed in a wide variety of applications, particular in connection with space heating and space cooling (see for example Ref. [2]). As yet it has not been used for electrical energy storage, but two technologies employing thermal reservoirs are currently under development, these being Advanced Adiabatic CAES (AA-CAES) and a scheme that will 
be referred to here as pumped thermal electricity storage or PTES. A full description of AA-CAES is given by Bullough et al [3], its main feature being that thermal energy is extracted and stored separately before the air enters the storage cavern. This increases the storage density and efficiency, whilst also protecting the cavern walls against exposure to high temperatures.

A simplified layout of a PTES scheme (shown during the charging phase) is given in Fig. 1. This interesting scheme seems to have been proposed almost simultaneously and independently in England [4] and France [5]. Effectively it operates as a reverse Joule (or Brayton) cycle heat pump during the charging phase, extracting heat from the cold reservoir and delivering heat to the hot reservoir. During discharge the processes are reversed such that the device operates as a closed Joule cycle heat engine. The version of PTES described in Ref. [5] uses turbomachinery for the compression and expansion processes, whereas that described in [4] employs reciprocating devices on the grounds that these might be made to achieve higher isentropic efficiencies. The two heat exchangers serve to reject heat at low temperature, this being necessary to counter the effects of system irreversibilities. Compared with AA-CAES, PTES has the advantage of higher storage density and it does not suffer the same geographical constraints. The highest pressures in a PTES system are also considerably lower than for CAES, thereby reducing the cost of the thermal storage containment vessel. However, a complete charge and discharge cycle involves twice as many compression, expansion and heat transfer processes, each of which entrain a degree of irreversibility.

The present paper focuses on the thermodynamic efficiency of the storage process within the thermal reservoirs alone. Losses within these reservoirs have two components: thermal losses (associated chiefly with irreversible heat transfer) and pressure losses. The latter are very straightforward and are readily computed, provided skin friction correlations are available for flow over the storage media. Thermal losses are less straightforward, and indeed their mag- 
nitude is likely to depend on the mode of operation of the storage scheme. Two limiting cases may be identified: (a) regular periodic charge and discharge (e.g., for diurnal load-levelling applications) and (b) longer term storage to cover unexpected shortfalls in electricity supply (e.g., those due to extended wind lull).

There is of course an extensive literature relating to thermal storage and regenerators. A comprehensive description of design and analysis techniques is given by Schmidt and Willmott [6] but, as noted by Krane [7] these techniques are based on First Law considerations. For electricity storage it is clearly the loss in availability associated with the storage process that is of relevance and so Second Law analysis is required. Krane, following Bejan's approach [8], undertook such an analysis for a "sensible heat" storage device, concluding that between 70 and $90 \%$ of the availability would be destroyed in a typical charge-discharge cycle. However, the device considered employed a liquid storage medium for which much of the availability is destroyed by irreversible mixing; solid media regenerators such as pebble beds potentially have much lower losses.

Regenerator performance has also been extensively treated by the Stirling engine fraternity and there are numerous relevant publications available in this field (see, for example, [9]). Nonetheless, the issues of concern to largescale electricity storage are sufficiently different as to warrant the separate treatment given in the present paper.

\section{NOTATION}
$A \quad$ reservoir cross-sectional area, $\left[\mathrm{m}^{2}\right]$
$B \quad$ stored availability (see eq.(30)), [J]
Bi Biot number, $h / k_{s} S_{v}$ 
$\mathrm{C}_{\mathrm{f}} \quad$ friction coefficient

$c_{p g} \quad$ gas isobaric specific heat capacity, $[\mathrm{J} / \mathrm{kgK}]$

$c_{s} \quad$ solid specific heat capacity, $[\mathrm{J} / \mathrm{kgK}]$

$d_{p} \quad$ particle diameter in packed bed, $[\mathrm{m}]$

$h \quad$ heat transfer coefficient, $\left[\mathrm{W} / \mathrm{m}^{2} \mathrm{~K}\right]$

$k \quad$ thermal conductivity, $[\mathrm{W} / \mathrm{mK}]$

$L \quad$ reservoir length, $[\mathrm{m}]$

$\ell \quad$ thermal length scale, $[\mathrm{m}]$

$\dot{m} \quad$ gas mass flow rate, $[\mathrm{kg} / \mathrm{s}]$

$M_{s} \quad$ total mass of solid storage medium, [kg]

$\mathrm{Nu} \quad$ Nusselt number, $h d_{p} / k_{g}$

$p \quad$ gas pressure, $\left[\mathrm{N} / \mathrm{m}^{2}\right]$

$\mathrm{Re}_{\mathrm{m}} \quad$ modified Reynolds number, $\rho_{g} u_{s} /(1-\epsilon) S_{v} \mu_{g}$

$\mathrm{Re}_{\mathrm{p}} \quad$ particle Reynolds number, $\rho_{g} u_{s} d_{p} / \mu_{g}$

St Stanton number, $h / \rho_{g} u_{s} c_{p g}$

$S_{v} \quad$ particle surface-to-volume ratio, $S_{v}=6 / d_{p}\left[\mathrm{~m}^{2} / \mathrm{m}^{3}\right]$

$T$ temperature $[\mathrm{K}]$

$t_{\mathrm{C}} \quad$ charge period during cyclic operation $[\mathrm{s}]$

$t_{\mathrm{N}}$ nominal (full) charge time $[\mathrm{s}]$

$u_{i} \quad$ interstitial velocity, $u_{s} / \epsilon[\mathrm{m} / \mathrm{s}]$

$u_{s} \quad$ superficial (empty tube) velocity, $\dot{m} / \rho_{g} A[\mathrm{~m} / \mathrm{s}]$

$V \quad$ reservoir (storage medium) volume, $\left[\mathrm{m}^{3}\right]$

$V_{\mathrm{N}} \quad$ nominal thermal front velocity, $[\mathrm{m} / \mathrm{s}]$

$\alpha \quad$ storage utilisation factor, $t_{\mathrm{C}} / t_{\mathrm{N}}$

$\beta \quad$ normalised availability (see eq.(4))

$\gamma \quad$ isentropic index

$\delta \quad$ dimensionless temperature difference, $\Delta T / T_{2}$

$\Delta T \quad$ temperature difference across reservoir, $T_{1}-T_{2}[\mathrm{~K}]$

$\epsilon \quad$ packed bed void ratio 
$\eta \quad$ dimensionless time variable

$\theta$ dimensionless temperature variable

$\Lambda \quad$ dimensionless reservoir length, $L / \ell$

$\phi \quad$ dimensionless exit temperature, $T_{2} / T_{0}$

$\Pi \quad$ dimensionless charge period, $t_{\mathrm{C}} / \tau$

$\rho \quad$ density, $\left[\mathrm{kg} / \mathrm{m}^{3}\right]$

$\tau \quad$ thermal time scale [s]

$\xi \quad$ dimensionless distance variable

$\zeta \quad$ loss coefficient (defined in appendix B)

\section{subscripts}

0,1,2 ambient, inlet (charged), exit (discharged)

$g, s \quad$ gas, solid

\section{BASIC DEFINITIONS AND STORAGE DENSITY}

The reservoirs of interest here exploit 'sensible heat' storage as opposed to latent heat of phase-change or heat of reaction. This allows integration with the two storage technologies discussed above without creating 'pinch point' problems. Figure 2 shows a schematic view of heat transfer within a thermal reservoir, together with typical axial temperature profiles. The reservoir comprises a solid storage medium which may be in the form of a packed bed (e.g., gravel or pebbles) or may be arranged so as to provide a uniform array of channels. The solid occupies a volumetric fraction $(1-\epsilon)$ of the reservoir and is held within a perfectly insulated containment vessel which may or may not be pressurised. The reservoir (a hot reservoir in the case shown) is arranged vertically in order to reduce buoyancy-driven mixing. During the charge phase, gas (typically air or Argon) enters the top of the reservoir with a mass flow 
rate of $\dot{m}$ and an initial temperature $T_{1}$, and is cooled by the storage medium which is initially at $T_{2}$. During discharge, the flow is reversed, entering from the bottom at temperature $T_{2}$. Note that cold reservoirs (e.g., CS in Fig. 1) will have $T_{1}$ below $T_{2}$, and cold gas will enter from the bottom during charge.

An ideal reservoir would have no thermal resistance between the gas and solid, and the thermal front would remain as an abrupt change in temperature from $T_{1}$ to $T_{2}$ as it progressed through the reservoir. Straightforward energy balance gives the speed of this front as:

$$
V_{\mathrm{N}}=\frac{\dot{m} c_{p g}}{A(1-\epsilon) c_{s} \rho_{s}}=\frac{\rho_{g} c_{p g}}{(1-\epsilon) \rho_{s} c_{s}} u_{\mathrm{s}}
$$

where $A$ is the (total) cross-sectional area of the reservoir, $\rho_{s}$ and $\rho_{g}$ are the solid and gas densities respectively, $c_{p g}$ and $c_{s}$ are the gas (isobaric) and solid (isochoric) specific heat capacities respectively, and $u_{\mathrm{s}}$ is the superficial (or open tube) gas velocity. In practice, the thermal front will not, of course, remain perfectly abrupt, but will instead spread out during its progress. Nonetheless, $V_{\mathrm{N}}$ may still be interpreted as the nominal speed of the front and the nominal charge time is thus given by:

$$
t_{\mathrm{N}}=\frac{L}{V_{\mathrm{N}}}=\frac{M_{s} c_{s}}{\dot{m} c_{p g}}
$$

where $L$ is the reservoir length and $M_{s}$ the mass of solid storage media.

\subsection{Energy Storage Density}

Ignoring the (usually) very small contribution from the gas, the change in internal energy of the reservoir between its fully discharged and fully charged states is simply $E_{\max }=M_{s} c_{s} \Delta T$. However, for electricity storage applications it is really the thermodynamic availability, that is relevant, this being the maximum work that can be extracted via an ideal heat engine rejecting heat to the environment at temperature $T_{0}$. In terms of a storage density (in $\mathrm{J} / \mathrm{m}^{3}$ ) 
the maximum availability (relative to the discharged state) is given by:

$$
\rho_{\mathrm{B}}=\frac{B_{\max }}{V}=(1-\epsilon) \rho_{s} c_{s}\left\{\Delta T-T_{0} \ln \left(\frac{T_{1}}{T_{2}}\right)\right\}
$$

where $V=A L$ is the reservoir volume. It is also useful to define a dimensionless availability which, in terms of the dimensionless temperature drop $\delta=\Delta T / T_{2}$ and the dimensionless exit temperature, $\phi=T_{2} / T_{0}$, is given by:

$$
\beta=\frac{B_{\max }}{M_{s} c_{s} T_{0}}=\delta \phi-\ln (1+\delta)
$$

Typical values of storage density are given in Table 1 compared with storage densities for CAES and PHS. For reasons discussed below, the full storage capacity cannot be exploited but the table nonetheless suggests that thermal reservoirs have the potential to provide relatively compact storage devices.

\section{THERMAL LOSSES}

Within the thermal front, the temperature difference between the gas and the solid (shown exaggerated in Fig. 2) results in irreversible heat transfer which is the main source of the thermal loss. This manifests itself as a smearing of the thermal front as it progresses through the reservoir, the consequences of which are two-fold:

(1) the availability of the heated solid at any time is less than would be the case were an abrupt thermal front to be maintained;

(2) the reservoir cannot be fully charged without hot gas issuing from the exit.

These two issues are theoretically quite separate, but in practice both impinge upon the thermodynamic efficiency of the storage process. 


\subsection{Governing Equations}

The equations governing heat exchange within thermal reservoirs have been presented many times within the literature (e.g., [10]) but are outlined here for completeness. The following simplifying assumptions are made (which are effectively equivalent to the so-called Schumann model):

(1) The reservoir is adiabatic and contains no radial or circumferential temperature variations. For the applications under consideration, it is obviously desirable that heat losses from the reservoirs are kept small. If this is achieved then radial temperature variations should also be small. Thus, although two-dimensional models may be found in the literature (e.g., $[11,12])$ these are usually aimed at applications where heat transfer at the wall is intentional.

(2) Heat transfer to and from the solid is limited by the thermal resistance at its surface (i.e., the Biot number is assumed zero). Estimates presented in section 5 suggests that this is justified.

(3) Conduction along the reservoir in both the gas and solid is neglected. Willmott [10] has presented numerical results of temperature distributions including the effects of longitudinal conduction which show that these effects are not dramatic, although neither are they insignificant. (Note that, in common with most models, radiative heat transfer has also been neglected here.)

With these assumptions, application of the unsteady flow energy equation to the gas and the First Law to the solid gives:

$$
\begin{gathered}
\dot{m} c_{p g} \frac{\partial T_{g}}{\partial x}+A \epsilon\left(\rho_{g} c_{p g} \frac{\partial T_{g}}{\partial t}-\frac{\partial p}{\partial t}\right)=h A(1-\epsilon) S_{v}\left(T_{s}-T_{g}\right) \\
\rho_{s} A(1-\epsilon) c_{s} \frac{\partial T_{s}}{\partial t}=h A(1-\epsilon) S_{v}\left(T_{g}-T_{s}\right)
\end{gathered}
$$


where $h$ is the surface heat transfer coefficient, $S_{v}$ is the solid surface area to volume ratio, and other symbols are as defined in the notation. (Note that the unsteady mass continuity equation has also been used in the derivation of eq.(5).) Accurate simulations require numerical integration of these equations together with the mass continuity and momentum equations in order to take account of gas density and other property variations. Although quite straightforward, there is not much to be gained from this extra complication and a clearer exposition is obtained by assuming average values for the gas density etc. and a constant mass flow rate. Neglecting the unsteady pressure term, eqs. (5) and (6) may then be expressed in the normalised form (see Ref. [10]):

$$
\begin{aligned}
& \frac{\partial \theta_{g}}{\partial \xi}=\theta_{s}-\theta_{g} \\
& \frac{\partial \theta_{s}}{\partial \eta}=\theta_{g}-\theta_{s}
\end{aligned}
$$

where $\theta_{g, s}=\left(T_{g, s}-T_{2}\right) / \Delta T$, and $\xi$ and $\eta$ are the dimensionless length and time variables given by:

$$
\xi=\frac{x}{\ell} \quad ; \quad \eta=\frac{t}{\tau}-\frac{\epsilon x}{u_{s} \tau}
$$

The length and time scales used for this normalisation are, respectively:

$$
\begin{gathered}
\ell=\frac{\dot{m} c_{p g}}{h A(1-\epsilon) S_{v}}=\frac{1}{\operatorname{St}(1-\epsilon) S_{v}} \\
\tau=\frac{\rho_{s} c_{s}}{h S_{v}}=\frac{\ell}{V_{\mathrm{N}}}
\end{gathered}
$$

where St $=h / \rho_{g} u_{s} c_{p g}$ is the Stanton number. Note that the dependence of $\eta$ on $x$ stems from the unsteady accumulation of internal energy within the gas and can be ignored due the low heat capacity of the gas per unit volume compared with that of the solid. 


\subsection{Single Charge Operation}

One possible use of an electricity storage device is to deal with infrequent and unexpected shortfalls in supply. In this case, the storage is likely to be long-term and the charging process for the reservoir is similar to the so-called single blow problem. An analytical solution to this problem was first presented in 1926 by Anzelius [13] and is described in detail in Ref. [10]. The result for the normalised temperature difference between the gas and the solid (starting from a fully discharged state at $\eta=0$ ) is:

$$
\theta_{g}-\theta_{s}=\exp \{-(\xi+\eta)\} I_{o}(2 \sqrt{\xi \eta})
$$

where $I_{o}$ is the zero-th order modified Bessel function of the first kind. As will become apparent, reduction of thermal losses requires the dimensionless reservoir length, $\Lambda=L / \ell$, and charge time, $\eta$, to be very large. Evaluation of the analytical solution then becomes cumbersome due to the divergence of $I_{o}$, and it is quicker and more reliable to integrate eqs. (7) and (8) numerically. An efficient numerical integration routine for this purpose is outlined in appendix A.

Computed gas temperature profiles are plotted in Fig. 3 and show how the thermal front becomes progressively less steep as it moves through the reservoir. The figure also shows curves of $\left(\theta_{g}-\theta_{s}\right)^{2}$ (dashed lines) which provide an estimate of the local rate of entropy increase due to irreversible heat transfer (see appendix B). The analytical solution for this quantity is shown for $\eta=10$ and demonstrates the accuracy of the numerical method.

Thermal Loss Coefficients Loss coefficients are defined in the present work as the loss in availability associated with each irreversible process, normalised with respect to the availability that has entered the reservoir. Ultimately it is the total loss for a complete charge-discharge cycle that is impor- 
tant, but it is nonetheless instructive to compute loss coefficients for a single charge / storage event. In this context there are three sources of thermal loss:

(1) The thermodynamic loss, quantified by $\zeta_{t}$, stems from irreversible heat transfer between the hot gas and cooler solid and is computed by integrating the entropy generation rate with respect to time.

(2) The exit loss, quantified by $\zeta_{\mathrm{x}}$, is due to hot gas issuing from the reservoir once it is almost fully charged. In principle the available energy carried by this gas could be exploited, but in most cases it is not practicable to do so. The sum of the exit and thermodynamic loss is determined by computing the available work stored within the solid and subtracting from the incoming availability.

(3) The storage loss is due to the levelling off of residual temperature gradients within the solid prior to discharge. The extent of this loss clearly depends on the storage duration but it reaches a maximum, quantified by $\zeta_{\mathrm{s} \infty}$, when all gradients have decayed. $\zeta_{\mathrm{s} \infty}$ can thus be determined by computing the stored availability at the final uniform temperature.

Expressions for the three loss coefficients are derived in appendix B and are plotted as a function of $\eta$ in Fig. 4 for reservoirs of dimensionless lengths $\Lambda=300$ and 600 , with $\delta=2$ and $\phi=1$ (i.e., the discharge state at ambient temperature). From this figure it is clear that the thermodynamic component of loss decreases continuously with $\eta$. This is because the gradient of the thermal front becomes progressively less steep and so heat transfer takes place over an increasing gas-solid interface. Reducing $\zeta_{t}$ therefore requires making the dimensionless length of the reservoir as long as possible. The exit loss is initially zero but rises steeply as the nose of the thermal front breaks through the exit - in this case just before $\eta=\Lambda$. Finally, the storage loss is initially very high but reduces rapidly as the reservoir reaches full charge since the average temperature of the storage media then approaches $T_{1}$. The total loss (applicable in cases of long storage duration) therefore has a sharp minimum, 
located at points $\mathrm{A}$ and $\mathrm{B}$ for $\Lambda=300$ and $\Lambda=600$ respectively.

As noted in appendix B, $\zeta_{t}$ (which constitutes the major part of the total loss) depends on the dimensionless temperature difference, $\delta$, and the dimensionless exit temperature, $\phi$, as well as upon $\eta$ and $\Lambda$. However, with very little error, $\zeta_{t}$ may be factorised into a temperature-dependent term, $\mathrm{F}(\delta, \phi)$, and a geometric term, $\mathrm{I}(\eta, \Lambda) / \eta$. This enables selection of optimum temperature conditions independently of the detailed geometry. Such optimisation should of course be undertaken for the storage system as a whole, but it is of interest to examine how the losses and storage capacity are influenced by the operating temperatures. Figure 5 shows the function $\mathrm{F}(\delta, \phi)$ plotted against dimensionless availability, $\beta$, which is proportional to storage density. The bold curve in the figure corresponds to an ambient discharge temperature $(\phi=1)$ and shows losses decreasing with storage density (i.e., as the reservoir gets hotter) for hot reservoirs, but increasing with storage density (i.e., as the reservoir gets colder) for cold reservoirs. (This opposite behaviour arises from the inverse dependence of the entropy generation rate on the absolute temperatures $T_{s}$ and $T_{g}$, as shown by eq.(26) in appendix B.) The other curves $(\phi \neq 1)$ suggest that it may be possible to significantly reduce thermal losses by selecting exit (discharged state) temperatures above and below ambient for hot and cold reservoirs respectively. (This may also help to reduce the effect of irreversibility in the compression and expansion devices, as discussed in Ref. $[14].)^{*}$

\footnotetext{
* It is important to note that for a PTES system the hot and cold reservoir temperatures cannot be varied independently since compression and expansion temperature ratios (see Fig. 1) are approximately equal. For such systems it is probably more convenient to employ combined loss coefficients for both reservoirs.
} 


\subsection{Cyclic Operation}

For load-levelling applications, the operation of the thermal reservoirs is more akin to regular, periodic charge and discharge. Calculations for this type of operation are undertaken by applying the same numerical scheme (see appendix A) and treating flow reversal between charge and discharge by setting:

$$
\theta_{s}^{\prime}(\xi, 0)=\theta_{s}(\Lambda-\xi, \Pi)
$$

where $\Pi=t_{\mathrm{C}} / \tau$ is the normalised charge period and the prime denotes quantities during discharge. A similar expression is applied at the end of discharge, and gas inflow conditions are given by $\theta_{g}(0, \eta)=1$ (charge) and $\theta_{g}^{\prime}(0, \eta)=0$ (discharge). This is essentially the same approach as that presented in [10]. For simplicity, it is assumed that charge and discharge are of equal duration and that one follows immediately after the other. Furthermore, mass flow rates, specific heat capacities and heat transfer coefficients are assumed unchanged between charge and discharge so that $\Lambda=\Lambda^{\prime}$ and $\Pi=\Pi^{\prime}$. (In regenerator parlance, the operation is said to be balanced and symmetric.) Computations are started from an initial fully-discharged state and, after a transient phase, reach a steady-state periodic mode wherein consecutive cycles are identical.

The performance of the reservoir under this cyclic operation is governed to a large extent by the duration of the charge period compared to the nominal charge time, i.e., by the ratio $\alpha=t_{\mathrm{C}} / t_{\mathrm{N}}=\Pi / \Lambda$. The quantity $\alpha$ may be interpreted as a capacity or utilisation factor since the availability stored during $t_{\mathrm{C}}$ is $\sim \alpha B_{\max }$. Figure 6 shows computed gas temperature profiles at various stages during the charge phase for $\alpha=0.50$ and $\alpha=0.25$. Towards the end of the charge period (the curves labelled (c) in the figure) the gas exit temperature begins to rise above $T_{2}$ and, as with single-charge operation, this gives rise to an exit loss. Similarly, towards the end of discharge the temperature of the returned gas falls below $T_{1}$, but it is still usually possible to extract work 
from this slightly cooler gas. The overall thermal loss coefficient, assuming zero storage loss, is thus:

$$
\zeta_{\mathrm{t}}+\zeta_{\mathrm{x}}=\frac{B_{\mathrm{in}}-B_{\mathrm{out}}}{B_{\mathrm{in}}}
$$

where $B_{\text {in }}$ and $B_{\text {out }}$ are in the inflow and outflow of availability (both at the top of the reservoir shown in Fig. 2) during charge and discharge respectively. For example,

$$
B_{\text {out }}=\dot{m} c_{p g} \int_{\text {dis. }}\left\{T_{g}(0)-T_{2}-T_{0} \ln \left(\frac{T_{g}(0)}{T_{2}}\right)\right\} \mathrm{d} t,
$$

where the integral is over the discharge period and $T_{g}(0)$ denotes the value at $x=0$ (i.e., the top of the reservoir in Fig. 2). The exit loss during the charge period is given by a similar expression to eq.(14) but with $T_{g}(0)$ replaced by $T_{g}(L)$ and the integration carried out over the charge period.

The two loss components are shown in Fig. 7 as a function of $\alpha$ for $\Lambda=150$ and $\Lambda=300$. Both components clearly increase with $\alpha$ (particularly for $\alpha \gtrsim 0.5$ ) and decrease with $\Lambda$, but over much of the range it is the thermodynamic component that dominates. The increase of $\zeta_{\mathrm{t}}$ with $\alpha$ may be explained by returning to Fig. 6 which (as would be expected) shows a steeper temperature gradient for the higher value of $\alpha$. As with the single-charge case, this means that heat transfer occurs over a smaller interfacial area resulting in a larger temperature drop between gas and solid and hence a higher entropy generation rate. In the limiting case of very short duration cycles $(\alpha \rightarrow 0)$, and provided the reservoir is sufficiently long that end effects may be neglected $(\Lambda \gg 1)$, the temperature profiles must ultimately tend towards the straight line distribution:

$$
\theta_{s} \simeq \theta_{g}=1-\xi / \Lambda
$$

From eq.(7) the temperature difference $\Delta \theta$ is then constant at $1 / \Lambda$ and so the expression for the entropy generation rate (eq.(26) in appendix B) may be integrated analytically. The resulting loss coefficient is given (after straight- 
forward manipulation) by:

$$
\zeta_{\mathrm{t}, \min }=\frac{2 \delta^{2}}{\Lambda(1+\delta)\{\delta \phi-\ln (1+\delta)\}}
$$

Values calculated from this expression are shown in Fig. 7 and agree very well with the numerical results. More generally, the inverse relation between $\zeta_{\mathrm{t}}$ and $\Lambda$ shown by eq.(16) appears to be approximately valid for values of $\alpha$ significantly above zero. This is supported by the fact that doubling $\zeta_{t}$ for $\Lambda=300$ agrees tolerably well with the curve for $\Lambda=150$ for $\alpha \lesssim 0.5$, as shown by the solid symbols in Fig. 7 .

\section{PRESSURE LOSSES}

The analysis of pressure losses within packed beds or other types of thermal reservoir is straightforward and can be found in many standard texts (see, for example, Ref. [15]). Without loss of generality, the pressure gradient may be written as:

$$
\frac{\partial p}{\partial x}=-\frac{1}{2}\left(\left\{\rho_{g} u_{s}\right\}^{2} S_{v} \frac{1-\epsilon}{\epsilon^{3}}\right) \frac{\mathrm{C}_{\mathrm{f}}}{\rho_{g}},
$$

where $\mathrm{C}_{\mathrm{f}}$ is the friction coefficient, which depends on the Reynolds number and geometric arrangement of the packing. The bracketed term may be treated as constant but the ratio $\left(\mathrm{C}_{\mathrm{f}} / \rho_{\mathrm{g}}\right)$ depends on gas temperature and hence varies over the length of the reservoir. As before, precise calculations therefore require eq.(17) to be integrated in conjunction with the energy equations $(5,6)$ and the mass continuity equation, but a reasonable estimate of the overall pressure loss, $\Delta p$, can be obtained by using an average value for $\left(\mathrm{C}_{\mathrm{f}} / \rho_{g}\right)$. In keeping with the thermal loss analysis, this pressure loss is expressed here a loss coefficient (i.e., fractional loss of availability), given by:

$$
\zeta_{\mathrm{p}}=\frac{T_{0} \Delta s_{\mathrm{p}}}{c_{p g} T_{0} \beta},
$$


where $\Delta s_{\mathrm{p}}$ is the gas specific entropy increase due to $\Delta p$. Provided the fractional pressure drop is small, $\Delta s_{\mathrm{p}} \approx R \Delta p / p$. Substituting into eq.(18) and making use of eq.(17) and eq.(9) then gives:

$$
\zeta_{\mathrm{p}}=\frac{\gamma-1}{2 \beta} M_{s}^{2}\left(\frac{1-\epsilon}{\epsilon^{3}}\right) \mathrm{C}_{\mathrm{f}} S_{v} L
$$

where $M_{s}$ is the Mach number based on $u_{s}$. As would be expected, $\zeta_{\mathrm{p}}$ increases with reservoir length and with the surface-to-volume ratio of the storage medium, so there is clearly a conflict between reducing the thermal and pressure losses. Mach numbers are usually very low, but high values of the

product $S_{v} L$ nonetheless mean that pressure losses may become significant, particularly for cold reservoirs where $M_{s}$ is greatest.

\section{DIMENSIONAL CALCULATIONS}

In the interests of generality, results thus far have been presented in terms of non-dimensional quantities such as $\Lambda, \Pi$ and $\beta$. However, the relationships between such quantities and the parameters that may be freely varied during the design process are not always transparent. For example, an energy storage installation is likely to be specified in terms of its energy storage capacity and power rating. This effectively sets the storage volume and gas mass flow rate (assuming the storage material, working fluid and cycle temperatures have also been specified). The main reservoir parameters remaining under the designer's control are then $L$ (or equivalently $A$ ), $S_{v}$ and possibly $\epsilon$, but these do not relate in a straightforward manner to the various loss coefficients, particularly because losses depend on skin friction and Stanton numbers. The purpose of the present section is thus to provide an estimate of the overall fractional loss in availability associated with the storage process for a nominal design of PTES installation. Consideration is then given to how losses might be minimised by adjustment of the design parameters. 
Table 2 gives the outline geometry and operating parameters for a nominal 2 MW PTES scheme with a maximum storage capacity of $\sim 12$ MWh, using Argon as the working fluid and gravel as the storage medium. The choice of optimum operating pressures and temperatures involves considerations (e.g., stressing, economics, cycle performance) beyond the scope of the present paper and so the set of values given in the table, although not unrealistic, serves only to provide an example.

Estimates are first required for $\mathrm{C}_{\mathrm{f}}$ and $\mathrm{St}$. The friction coefficient is obtained from the Carman correlation [15]:

$$
\mathrm{C}_{\mathrm{f}}=\frac{10}{\operatorname{Re}_{\mathrm{m}}}+\frac{8}{10 \operatorname{Re}_{\mathrm{m}}{ }^{1 / 10}}
$$

where $\mathrm{Re}_{\mathrm{m}}$ is the modified Reynolds number, as defined in the notation. Estimation of the Stanton number is subject to considerable uncertainty due to the difficulties in obtaining reliable measurements of heat transfer coefficients in packed beds - see, for example, the discussions in Refs. [16,17]. For the range of Reynolds number of interest, Wakao et al [17] suggest the following Nusselt number correlation:

$$
\mathrm{Nu}=2.0+1.10 \operatorname{Pr}^{1 / 3} \operatorname{Re}_{\mathrm{p}}^{3 / 5}
$$

where Pr is the Prandtl number and $\operatorname{Re}_{\mathrm{p}}=\rho_{\mathrm{g}} \mathrm{u}_{\mathrm{s}} \mathrm{d}_{\mathrm{p}} / \mu_{\mathrm{g}}$ is the particle Reynolds number. It is worth noting that the experimental data presented in Ref. [17] straddle the above correlation and encompass a factor of $\sim 4$ in the range of interest. (The data are for spherical particles for which $S_{v}=6 / d_{p}$.)

Table 3 shows the the main dimensionless parameters together with estimates for the various loss coefficients for the hot and cold reservoirs. (Note that the estimated Biot numbers are very low so that thermal resistance is dominated by the solid-gas surface term and the lumped-capacity model is therefore justified.) For single-charge operation the values of the $\zeta$ 's given in the table are for the charging process alone. Similar pressure and thermodynamic losses 
would occur during discharge ${ }^{\dagger}$, but the discharge exit loss cannot easily be quantified without regard for the other components in the cycle: for example, at some point the discharge temperature of the hot reservoir will fall below a value at which it is practical to extract work from the turbine. For simplicity, the exit loss during discharge is assumed equal to that during charge, giving:

$$
\zeta_{\text {tot }}^{\text {sin. }}=2\left(\zeta_{\mathrm{t}}+\zeta_{\mathrm{x}}+\zeta_{\mathrm{p}}\right)+\zeta_{\mathrm{s} \infty}
$$

Tabulated values are those obtained by minimisation of $\zeta_{\text {tot }}^{\text {sin. }}$ in a fashion similar to that demonstrated in Fig. 4. For cyclic operation,

$$
\zeta_{\text {tot }}^{\text {cyc. }}=\zeta_{\mathrm{t}}+\zeta_{\mathrm{x}}+2 \zeta_{\mathrm{p}}
$$

The total losses (highlighted in bold in the table) are obtained by weighting values for the hot and cold reservoirs by their respective storage densities (or, equivalently, $\beta$ ).

It is notable from the table that both thermal and pressure loss coefficients are considerably greater for the cold reservoir than for the hot one. The lower pressure in the cold reservoir results in a lower gas density which in turn leads to higher velocities and a higher (absolute) pressure drop. Furthermore, it is really the fractional pressure drop that is relevant since the work that can be extracted by a turbine (or other expansion device) depends on the pressure ratio across it. Thus, in the cold (low pressure) reservoir, the pressure drop through the packing has a bigger impact. The higher thermal losses are due to the lower dimensionless length (which in turn is due to lower Stanton numbers at the higher Reynolds numbers) and higher value of $\mathrm{F}(\delta, \phi)$, as shown by points $\mathrm{H}$ and $\mathrm{C}$ in Fig. 5, corresponding to the hot and cold reservoir conditions respectively.

$\lceil$ This will be accurate if the storage is of long duration but will otherwise overestimate the thermodynamic loss since the returning thermal front during discharge is then less steep than during charge. 
It is also apparent that losses are significantly lower for periodic operation than for single charge due to the more gentle temperature gradients in the former. This is of course at the expense of reduced storage capacity. (On the basis of Fig. 7, a value of $\alpha=0.5$ was used to generate the results in Table 3, this providing a reasonable compromise between loss and utilisation.)

\subsection{Loss Minimisation}

Optimisation for an energy storage installation should be undertaken for the system as a whole, but it is nonetheless instructive to examine how loss for an individual reservoir might be minimised. As an example, Fig. 8 shows how the total loss (as given by eq.(22)) varies with reservoir length for a cold reservoir operated in a single charge-discharge mode. The curves shown have been computed for fixed mass flow and reservoir volume (i.e., fixed power and storage capacity) at two different particle sizes. Over the range of Reynolds numbers concerned, the friction coefficient remains approximately constant, so pressure losses scale as $\sim u_{s}^{2} L$. Increasing the length (at fixed volume) decreases the cross-sectional area and increases $u_{s}$, so that $\zeta_{\mathrm{p}}$ follows very closely a cubic variation with $L$. By contrast, all thermal loss components fall with length such that there is a minimum in $\zeta_{\text {tot }}^{\text {sin. }}$. For the hot reservoir, fractional pressure losses are much smaller, so the optimum shape is a longer, thinner reservoir. Choosing the correct geometry clearly has a significant impact, but in practice this requires more reliable estimates of heat transfer coefficient particular to the storage medium in use.

\section{CONCLUSION REMARKS}

An analysis of losses in thermal reservoirs due to irreversible heat transfer and viscous effects has been presented. The simplifying assumptions involved, 
particularly for the thermal analysis, mean that loss coefficients are likely to be underestimated since a number inherently irreversible processes have been neglected. However, the greatest source of uncertainty stems from the correlations employed for heat transfer and friction coefficients since there is considerable spread in the experimental data for these quantities. Despite these shortcomings, the following points may be concluded with reasonable confidence:

i. Together, thermal and viscous (pressure) losses are significant (typically upwards of a few percent for regular, periodic operation) but not so large as to preclude the possibility of using thermal reservoirs as part of an electricity storage scheme.

ii. Losses for one-off charge and discharge are substantially greater than for periodic operation due to the steeper thermal front associated with the former. This implies that long-term storage applications will suffer greater losses than, for example, diurnal load-levelling.

iii. Due to the essentially conflicting requirements of high surface area for heat transfer and low surface area for viscous loss, there is potential for substantial loss reduction by geometric optimisation.

iv. Thermal loss coefficients depend strongly on the operating temperatures in a manner that is not straightforward. This provides further opportunity for optimisation, but this should be undertaken for the system as a whole since the performance of the individual cycle components is inextricably linked.

\section{References}

[1] D. J. C. Mackay, Sustainable Energy without the Hot Air. UIT Cambridge Ltd., 2009 .

[2] A. M. khudhair and M. M. Farid, "A review of energy conservation in building 
applications with thermal storage by latent heat using phase change materials," Energy Conversion and Management, vol. 45, pp. 263-275, 2004.

[3] C. Bullough, C. Gatzen, C. Jakiel, M. Koller, A. Nowi, and S. Zunft, "Advanced adiabatic compressed air energy storage for the integration of wind energy," in Proc. of the European Wind Energy Conference EWEC, (London), 2004.

[4] J. Macnaghten and J. S. Howes, "Energy storage," tech. rep., International Patent No. WO 2009/044139 A2, 2009.

[5] T. Desrues, J. Ruer, P. Marty, and J. F. Fourmigué, "A thermal energy storage process for large scale electric applications," Applied Thermal Engineering, vol. 30 , pp. 425-432, 2010.

[6] F. W. Schmidt and A. J. Willmott, Thermal Energy Storage and Regeneration. Hemisphere Press, 1981.

[7] R. J. Krane, "A Second Law analysis of the optimum design and operation of thermal energy storage systems," Int. J. Heat Mass Trans., vol. 30, no. 1, pp. $43-57,1987$.

[8] A. Bejan, Entropy Generation Through Heat and Fluid Flow. John Wiley, 1982.

[9] A. J. Organ, The Regenerator and the Stirling Engine. London: Mechanical Engineering Publications, 1997.

[10] A. Willmott, Dynamics of Regenerative Heat Tranfer. Taylor \& Francis, 2002.

[11] A. P. De Wasch and G. F. Froment, "Heat transfer in packed beds," Chem. Eng. Sci., vol. 27, pp. 567-576, 1972.

[12] C. Li and B. A. Finlayson, "Heat transfer in packed beds - a reevaluation," Chem. Eng. Sci., vol. 32, pp. 1055-1066, 1977.

[13] A. Anzelius, "Heating by means of percolating media," J. of Mechanical Design, vol. 6, pp. 291-294, 1926.

[14] A. White, "Electricity storage using a thermal storage scheme," in 8th Int. Conf. of Comp. Methods in Sci. and Eng. (ICCMSE), (Kos, Greece), October 2010. 
[15] R. G. Holdich, Fundamentals of Particle Technology. Midland Information Technology and Publishing, 2002.

[16] H. Martin, "Low Peclet number particle-to-fluid heat and mass transfer in packed beds," Chem. Eng. Sci., vol. 33, pp. 913-919, 1978.

[17] N. Wakao, S. Kaguei, and T. Funazkri, "Effect of fluid dispersion coefficients on particle-to-fluid heat transfer coefficents in packed beds: correlation of Nusselt numbers," Chem. Eng. Sci., vol. 34, pp. 325-336, 1979.

\section{APPENDIX A: NUMERICAL INTEGRATION ROUTINE}

The numerical method used to integrate equations $(7 \& 8)$ is similar to that presented in Ref. [10], but stability and computational efficiency have been improved by employing a semi-analytical approach. Figure 9 shows part of the (regular) computational grid at two time levels. At a particular stage in the calculation, temperatures are known at all nodes for time step $n-1$ and for nodes up to and including $i-1$ at time step $n$. The task is therefore to determine temperatures at $(i, n)$. This is achieved by integrating eq.(7) analytically between nodes $i-1$ and $i$ whilst treating $\theta_{s}$ as a constant, giving:

$$
\theta_{g, i}^{n}=\bar{\theta}_{s}(1-\exp \{-\Delta \xi\})+\theta_{g, i-1}^{n} \exp \{-\Delta \xi\}
$$

where $\bar{\theta}_{s}$ is taken as the average value between $i-1$ and $i$ at time $n$. A similar integration is applied to eq.(8), taking a constant, average value for $\theta_{g}$ between $n-1$ and $n$ at node $i$. The resulting pair of equations may be expressed in the form:

$$
\left(\begin{array}{cc}
1 & \frac{1}{2}(a-1) \\
\frac{1}{2}(b-1) & 1
\end{array}\right)\left(\begin{array}{c}
\theta_{g, i}^{n} \\
\theta_{s, i}^{n}
\end{array}\right)=\left(\begin{array}{c}
\frac{1}{2}(1-a) \theta_{s, i-1}^{n}+a \theta_{g, i-1}^{n} \\
\frac{1}{2}(1-b) \theta_{g, i}^{n-1}+b \theta_{s, i}^{n-1}
\end{array}\right),
$$

where $a=\exp \{-\Delta \xi\}$ and $b=\exp \{-\Delta \eta\}$. Eq.(25) is readily inverted to obtain 
the two unknown temperatures. The advantage of this approach is that the 'stiffness' is integrated out of the equations enabling much larger time steps to be taken than with a straightforward finite difference discretisation.

\section{APPENDIX B: THERMAL LOSS COEFFICIENTS}

Loss coefficients are defined here as the fractional loss in availability associated with each of the loss-making processes. Derivations of the various thermal loss coefficients are given below for the single charge case. The unsteady accumulation of gas internal energy has been neglected in these derivations (see the note following eq.(10)). Since the heat capacity of the gas per unit storage volume is typically three orders of magnitude less than that of the solid, this effectively corresponds to over-predicting the thermodynamic loss by $\sim 0.1 \%$ of the stored availability.

\section{Thermodynamic Loss}

The major component of the thermal loss is that due to irreversible heat transfer and is determined by integrating the entropy generation rate over the length of the reservoir and over the duration of the charging process. The total, instantaneous entropy generation rate is given by:

$$
\begin{aligned}
\dot{S}_{\mathrm{irr}} & =\int\left(\frac{1}{T_{s}}-\frac{1}{T_{g}}\right) \mathrm{d} \dot{Q} \\
& =\int_{0}^{L}\left(\frac{1}{T_{s}}-\frac{1}{T_{g}}\right) h A(1-\epsilon) S_{v}\left(T_{g}-T_{s}\right) \mathrm{d} x,
\end{aligned}
$$

and the associated loss coefficient at time $t$ (starting from a fully discharged reservoir at $t=0)$ is:

$$
\zeta_{\mathrm{t}}=\frac{1}{\dot{m} c_{p g} T_{0} \beta t} \int_{0}^{t} T_{0} \dot{S}_{\mathrm{irr}} \mathrm{d} t
$$


Combining Eqs. (26) \& (27) and incorporating the expression for the thermal length scale, $\ell$, gives:

$$
\zeta_{\mathrm{t}}=\frac{\delta^{2}}{\beta \eta} \int_{0}^{\eta} \int_{0}^{\Lambda} \frac{\left(\theta_{g}-\theta_{s}\right)^{2}}{\left(1+\theta_{g} \delta\right)\left(1+\theta_{s} \delta\right)} \mathrm{d} \xi \mathrm{d} \eta
$$

The presence of $\delta$ in the integrand of this expression suggests that the temperature and geometric dependence of $\zeta_{\mathrm{t}}$ are inextricably linked. However, with reference to Fig. 3 it is reasonable to suppose that the major contribution to the integral in eq.(28) occurs for values of $\theta_{g}$ and $\theta_{s}$ of $\sim \frac{1}{2}$ so that the denominator in the integrand can be approximated by $\left(1+\delta+\delta^{2} / 4\right)$. In fact this tends to slightly underestimate the loss, but numerical experimentation shows that, with virtually no error (less than $0.25 \%$ over the ranges of $\delta$ and $\eta$ of relevance), eq.(28) may be replaced by:

$$
\zeta_{\mathrm{t}}=\frac{\delta^{2}}{\left(1+\delta+\delta^{2} / 12\right) \beta \eta} \int_{0}^{\eta} \int_{0}^{\Lambda}\left(\theta_{g}-\theta_{s}\right)^{2} \mathrm{~d} \xi \mathrm{d} \eta=\mathrm{F}(\delta, \phi) \frac{\mathrm{I}(\eta, \Lambda)}{\eta} .
$$

The significance of this is that the function $\mathrm{F}$ depends only on the operating temperatures, whereas $\mathrm{I}(\eta, \Lambda) / \eta$ depends only on geometric factors ${ }^{\dagger}$ and the level of charge. (Note that $\eta=\Lambda$ implies $t=t_{\mathrm{N}}$ and hence corresponds to nominal full charge.)

\section{Exit Loss}

This stems from hot gas (or cold gas in the case of cold storage) issuing from the reservoir exit as it approaches full charge. The availability stored within the reservoir at any time relative to its discharged state is:

$$
B(t)=\int_{0}^{L} \rho_{s} c_{s}(1-\epsilon) A\left\{\left(T_{s}-T_{2}\right)-T_{0} \ln \left(\frac{T_{s}}{T_{2}}\right)\right\} \mathrm{d} x
$$

$\bar{\dagger}$ In fact, as shown by the $\zeta_{\mathrm{t}}$ curve in Fig. 4, the integral is independent of $\Lambda$ until the thermal front breaks through the exit plane. 
whereas the maximum availability that could have been stored (in an infinite reservoir) in the same time is $B_{\mathrm{in}}=\dot{m} c_{p g} T_{0} \beta t$. The difference between these two quantities is the lost work associated with both irreversible heat transfer and the exit flow. Thus,

$$
\zeta_{\mathrm{t}}+\zeta_{\mathrm{x}}=1-\frac{B}{B_{\mathrm{in}}}=1-\frac{1}{\beta \eta} \int_{0}^{\Lambda}\left(\theta_{s} \delta \phi-\ln \left\{1+\theta_{s} \delta\right\}\right) \mathrm{d} \xi
$$

The exit loss is zero in the initial stages of charge (see Fig. 4) so eqs. (29) \& (31) should then yield identical results. This is used as a check that the grid and time steps are sufficiently small to provide accurate results.

\section{Storage Loss}

Once charged, any residual temperature gradients within the storage medium will gradually decay, leading to a further reduction in availability. The mechanism for this process (i.e., axial conduction through the reservoir) has not been included within the analysis, but the maximum value of the storage loss can nonetheless be computed. This maximum occurs when the storage medium has acquired a uniform temperature which, assuming the storage is adiabatic, is given by the average value:

$$
\bar{\theta}_{s}=\frac{1}{\Lambda} \int_{0}^{\Lambda} \theta_{s} \mathrm{~d} \xi
$$

The total loss during charge plus storage (but not discharge) is then:

$$
\zeta_{\text {tot }}=\zeta_{\mathrm{t}}+\zeta_{\mathrm{x}}+\zeta_{\mathrm{s} \infty}=1.0-\left(\bar{\theta}_{s} \delta \phi-\ln \left\{1+\bar{\theta}_{s} \delta\right\}\right) / \beta
$$

from which the maximum storage loss, $\zeta_{\mathrm{s} \infty}$, can be obtained by subtracting eq.(31). $\zeta_{\text {tot }}$ is a function of $\eta$ (see Fig. 4) because the average temperature, $\bar{\theta}_{s}$, clearly depends on the extent to which the reservoir is charged. 


\section{List of Tables}

1 Comparison of storage density, $\rho_{\mathrm{B}}$, for various technologies.

For the hot an cold reservoirs, the assumed material properties are $\rho_{s}(1-\epsilon)=1500 \mathrm{~kg} / \mathrm{m}^{3}$ and $c_{s}=800 \mathrm{~J} / \mathrm{kgK}$, and $T_{2}=T_{0}=300 \mathrm{~K}$

2 Geometry and operating conditions of the hot and cold reservoirs for a PTES system

3 Estimation of losses for the hot and cold reservoirs of Table 2 


\section{List of Figures}

1 Simplified layout for a pumped thermal electricity storage scheme shown during the charging phase. HX1 and HX2 are heat exchangers used to reject heat at near-ambient conditions. The compressor, C, and expander, E, may be turbomachines as in Ref. [5] or reciprocating devices, as in Ref. [4]

2 Schematic view of heat transfer within the reservoir and associated temperature profiles

3 Computed temperature profiles at three different times. The solid lines show the gas temperature, $\theta_{g}$, and the dashed lines show $\left(\theta_{g}-\theta_{s}\right)^{2}$ (right-hand axis). The analytical solution for the latter quantity has been plotted for $\eta=10$.

4 Computed loss coefficients as a function of $\eta$ for reservoirs with $\Lambda=300$ and $600, \Delta T=600 K$ and $T_{2}=T_{0}=300 K$.

5 Temperature dependence of the thermodynamic loss, $\mathrm{F}(\delta, \phi)$, plotted as a function of dimensionless availability, $\beta$, for different values of the exit temperature $\phi=T_{2} / T_{0}$. Solid and dashed curves are for hot and cold reservoirs respectively. The solid symbol corresponds to the same conditions as Fig. 4 and the points $\mathrm{H}$ and $\mathrm{C}$ correspond to the hot and cold reservoir conditions of section 5 .

6 Gas temperature profiles during cyclic operation with $\Lambda=300$. Above: period $\Pi=150$; Below: period $\Pi=75$. Curve: (a) Beginning of charge, $\eta=0.05 \Pi$; (b) Mid charge, $\eta=0.5 \Pi$; (c) End of charge, $\eta=0.95 \Pi$ 
7 Computed loss coefficients for cyclic operation as a function of utilisation factor, $\alpha$, with $T_{1}=600 \mathrm{~K}$ and $T_{2}=T_{0}=300 \mathrm{~K}$ $(\delta=2$ and $\phi=1)$.

8 Variation of total loss with reservoir length at fixed volume and mass flow rate for a cold reservoir operated in single charge-discharge mode. Conditions are as specified in Table 2, but with the reservoir length and diameter allowed to vary whilst keeping volume constant.

9 A section of the computational grid: temperatures are known at nodes marked $\bullet$ and unknown at nodes marked $\circ$. 


\section{TABLES}

\begin{tabular}{|l|c|c|c|c|}
\hline \multicolumn{2}{|l|}{ Technology / Storage } & Expression for $\rho_{\mathrm{B}}$ & Assumptions & $\rho_{\mathrm{B}}, \mathrm{MJ} / \mathrm{m}^{3}$ \\
\hline \hline PHS & Water & $\rho g \Delta H$ & $\Delta H=500 \mathrm{~m}$ & 5 \\
\hline CAES & Air & $p\left\{\ln \left(p / p_{0}\right)-\left(1-p_{0} / p\right)\right\}$ & $p=100 \mathrm{bar}$ & 36 \\
\hline Hot Res. & Gravel & Eq. $(3)$ & $T_{1}=773 \mathrm{~K}$ & 227 \\
\hline Cold Res. & Gravel & Eq. $(3)$ & $T_{1}=123 \mathrm{~K}$ & 109 \\
\hline
\end{tabular}

Table 1

Comparison of storage density, $\rho_{\mathrm{B}}$, for various technologies. For the hot an cold reservoirs, the assumed material properties are $\rho_{s}(1-\epsilon)=1500 \mathrm{~kg} / \mathrm{m}^{3}$ and $c_{s}=800$ $\mathrm{J} / \mathrm{kgK}$, and $T_{2}=T_{0}=300 \mathrm{~K}$ 


\begin{tabular}{|c|c|c|c|}
\hline & & Hot Resevoir & Cold Reservoir \\
\hline Inlet temperature (charge): & $T_{1}$ & $773 \mathrm{~K}$ & $123 \mathrm{~K}$ \\
\hline Exit temperature (charge): & $T_{2}$ & $300 \mathrm{~K}$ & $300 \mathrm{~K}$ \\
\hline Normalised availability: & $\beta$ & 0.627 & 0.301 \\
\hline Operating pressure: & $p$ & 10 bar & 1 bar \\
\hline Reservoir internal diameter: & $D$ & $5.0 \mathrm{~m}$ & $5.0 \mathrm{~m}$ \\
\hline Reservoir length: & $L$ & $5.0 \mathrm{~m}$ & $5.0 \mathrm{~m}$ \\
\hline \multicolumn{4}{|l|}{ Gravel storage medium: } \\
\hline Void fraction & $\epsilon$ & \multicolumn{2}{|c|}{0.33} \\
\hline Particle size & $d_{p}$ & \multicolumn{2}{|c|}{$0.02 \mathrm{~m}$} \\
\hline \multicolumn{4}{|l|}{ Argon working fluid: } \\
\hline Mass flow rate & $\dot{m}$ & \multicolumn{2}{|c|}{$12.5 \mathrm{~kg} / \mathrm{s}$} \\
\hline Nominal storage & $\Delta B$ & \multicolumn{2}{|c|}{$12 \mathrm{MWh}$} \\
\hline Nominal charge time & $t_{\mathrm{N}}$ & \multicolumn{2}{|c|}{$6 \mathrm{~h}$} \\
\hline Nominal power & $\dot{W}_{\text {net }}$ & \multicolumn{2}{|c|}{$2 \mathrm{MW}$} \\
\hline
\end{tabular}

Table 2

Geometry and operating conditions of the hot and cold reservoirs for a PTES system 


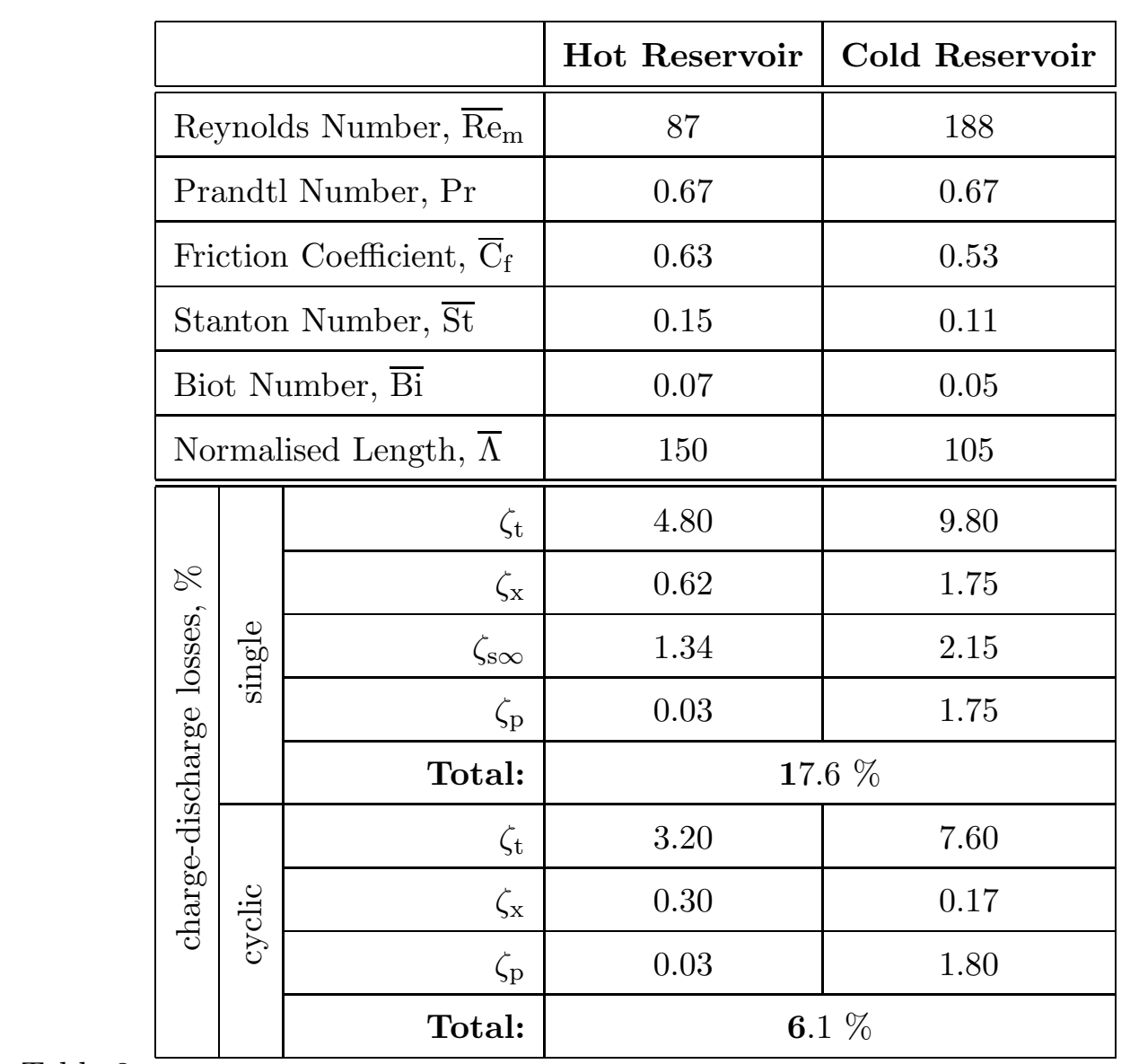

Table 3

Estimation of losses for the hot and cold reservoirs of Table 2. $(\alpha=0.5$ for the cyclic calculations.) 


\section{FIGURES}

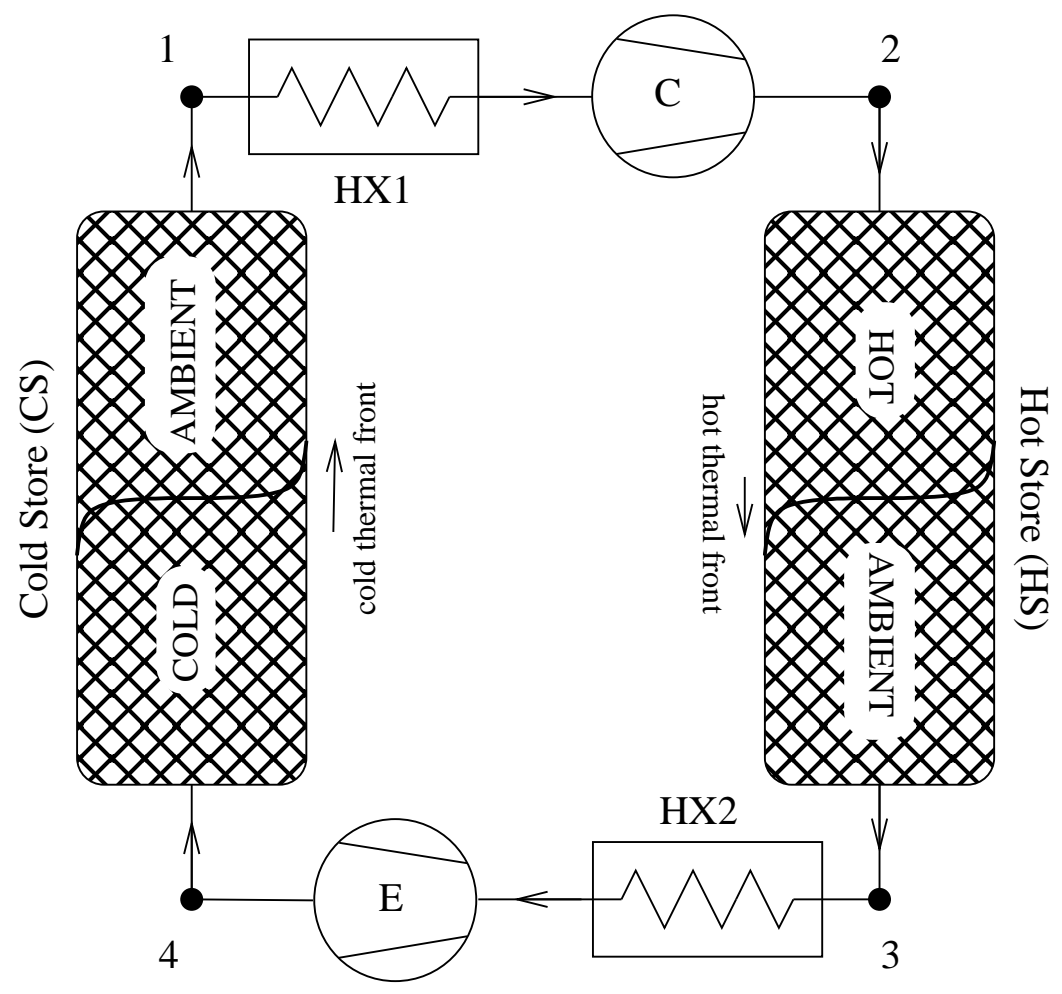

Figure 1. Simplified layout for a pumped thermal electricity storage scheme shown during the charging phase. HX1 and HX2 are heat exchangers used to reject heat at near-ambient conditions. The compressor, C, and expander, E, may be turbomachines as in Ref. [5] or reciprocating devices, as in Ref. [4] 

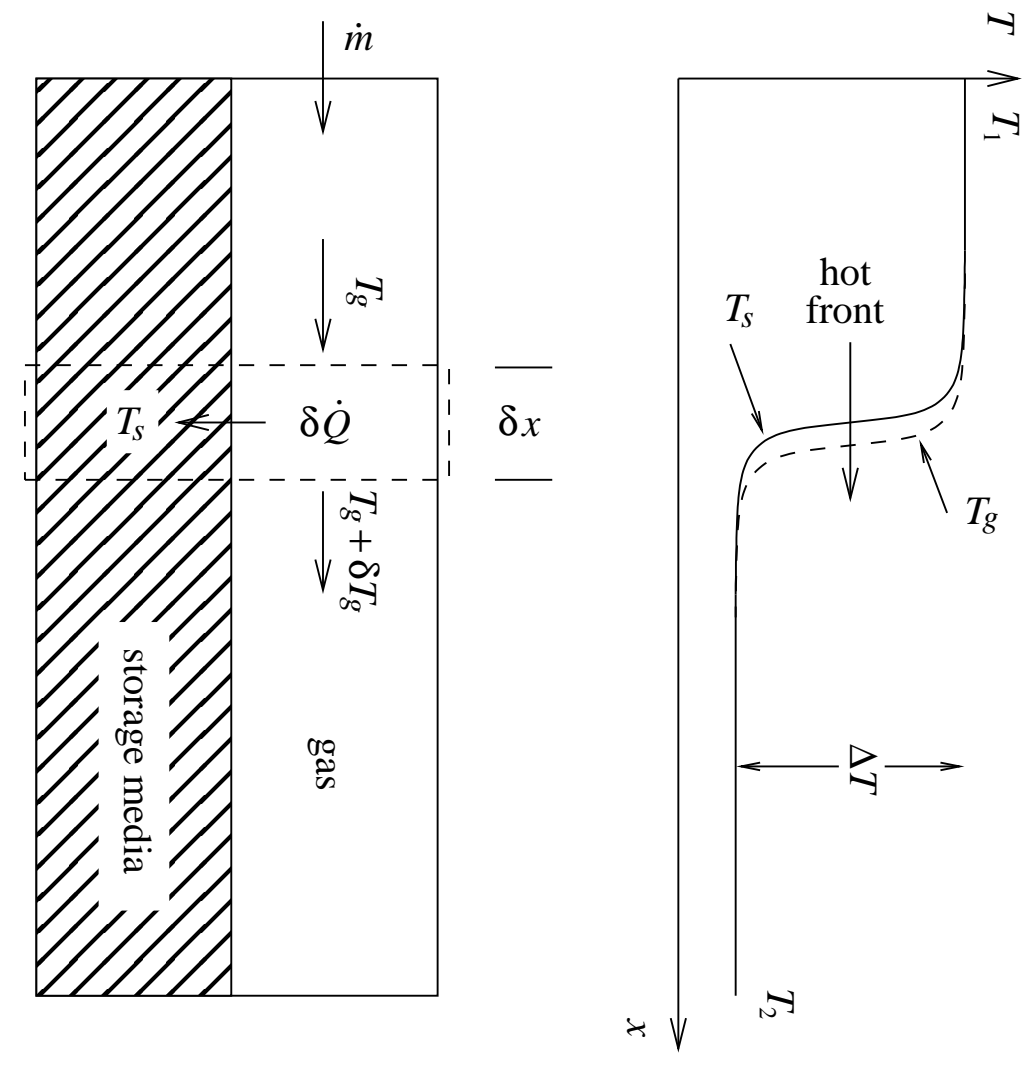

Figure 2. Schematic view of heat transfer within the reservoir and associated temperature profiles 


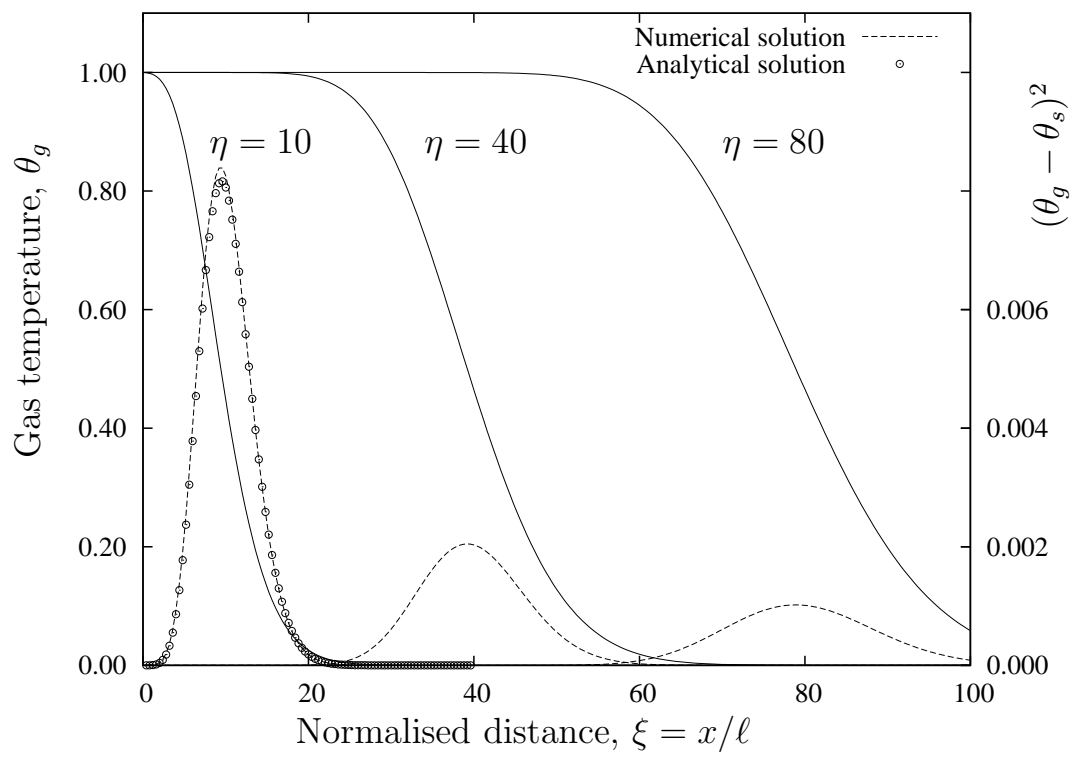

Figure 3. Computed temperature profiles at three different times. The solid lines show the gas temperature, $\theta_{g}$, and the dashed lines show $\left(\theta_{g}-\theta_{s}\right)^{2}$ (right-hand axis). The analytical solution for the latter quantity has been plotted for $\eta=10$. 


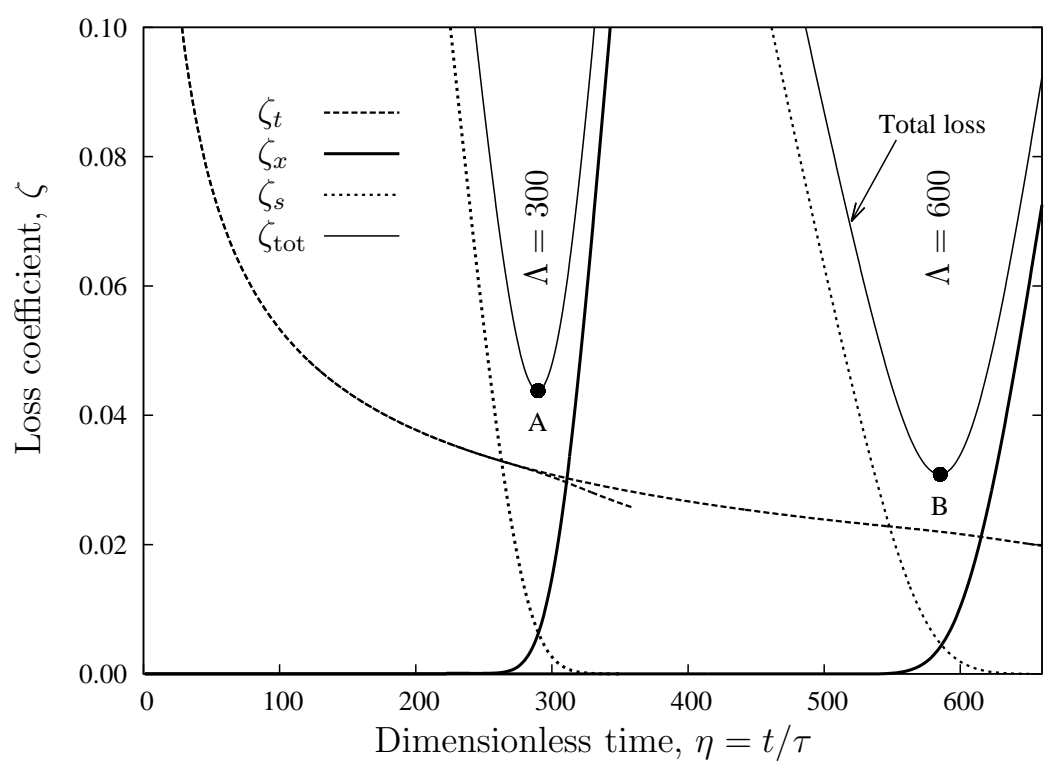

Figure 4. Computed loss coefficients as a function of $\eta$ for reservoirs with $\Lambda=300$ and $600, \Delta T=600 K$ and $T_{2}=T_{0}=300 K$. 


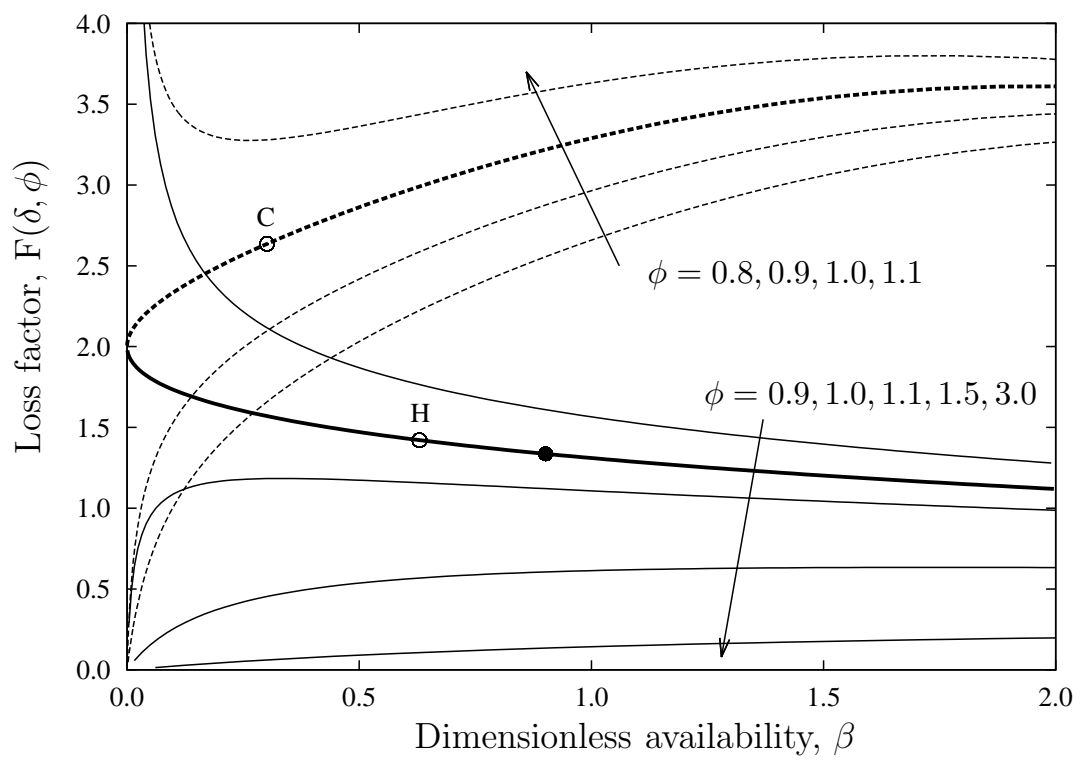

Figure 5. Temperature dependence of the thermodynamic loss, $\mathrm{F}(\delta, \phi)$, plotted as a function of dimensionless availability, $\beta$, for different values of the exit temperature $\phi=T_{2} / T_{0}$. Solid and dashed curves are for hot and cold reservoirs respectively. The solid symbol corresponds to the same conditions as Fig. 4 and the points $\mathrm{H}$ and $\mathrm{C}$ correspond to the hot and cold reservoir conditions of section 5. 
(i) $\Pi / \Lambda=0.50$

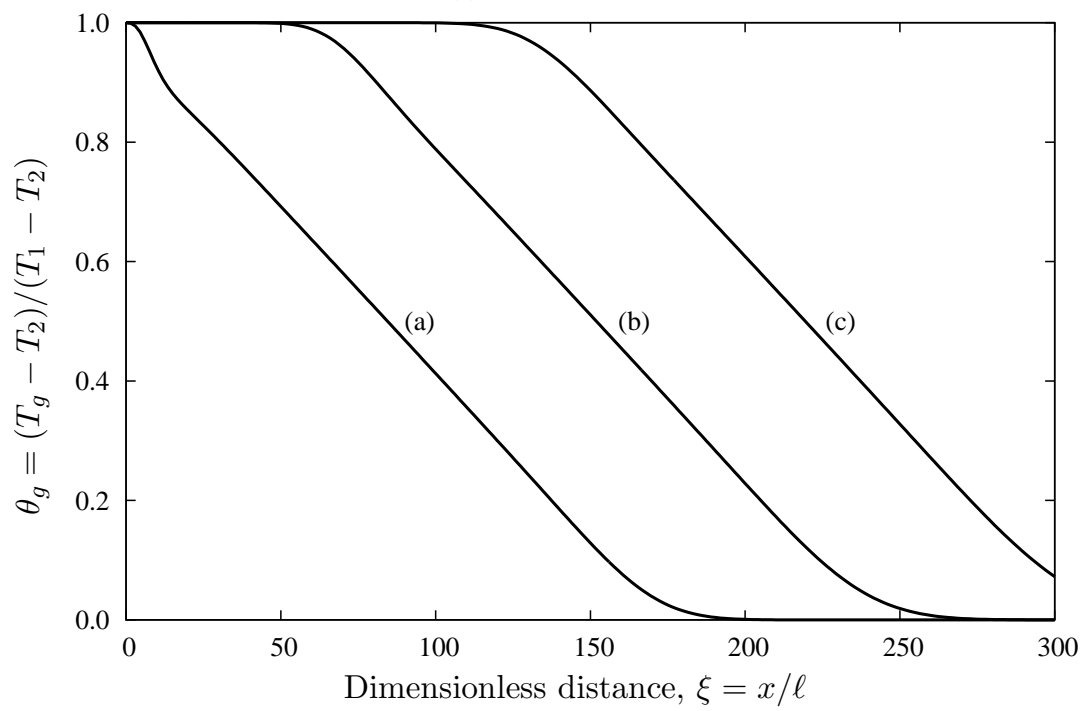

(ii) $\Pi / \Lambda=0.25$

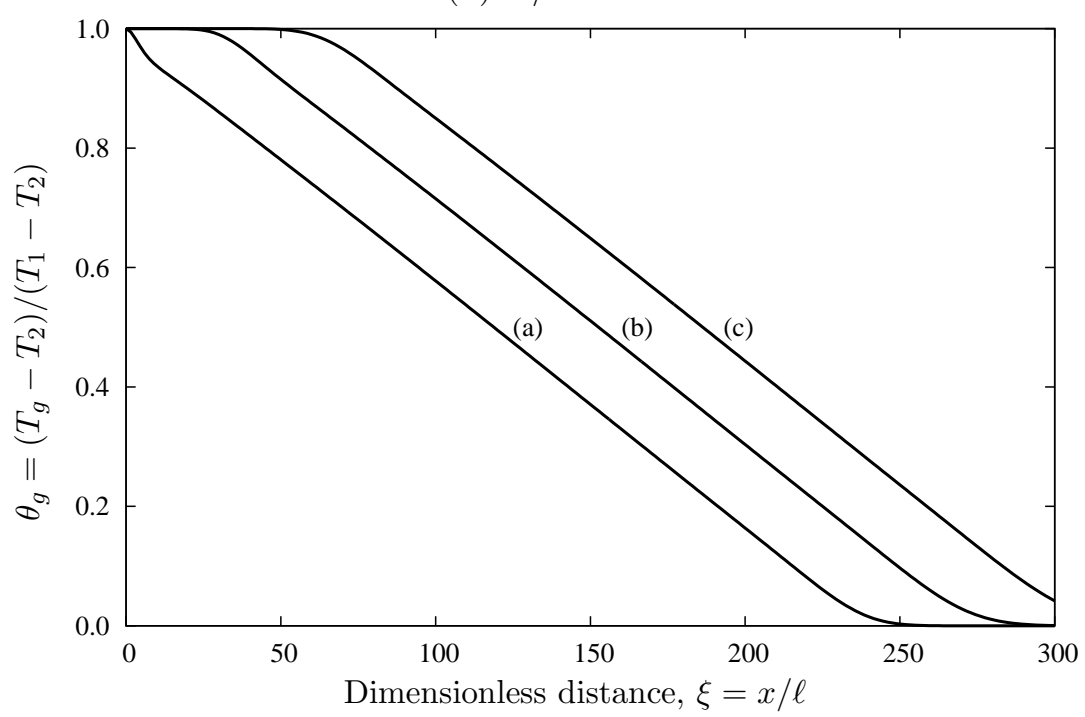

Figure 6. Gas temperature profiles during cyclic operation with $\Lambda=300$. Above: period $\Pi=150$; Below: period $\Pi=75$. Curve: (a) Beginning of charge, $\eta=0.05 \Pi$; (b) Mid charge, $\eta=0.5 \Pi$; (c) End of charge, $\eta=0.95 \Pi$ 


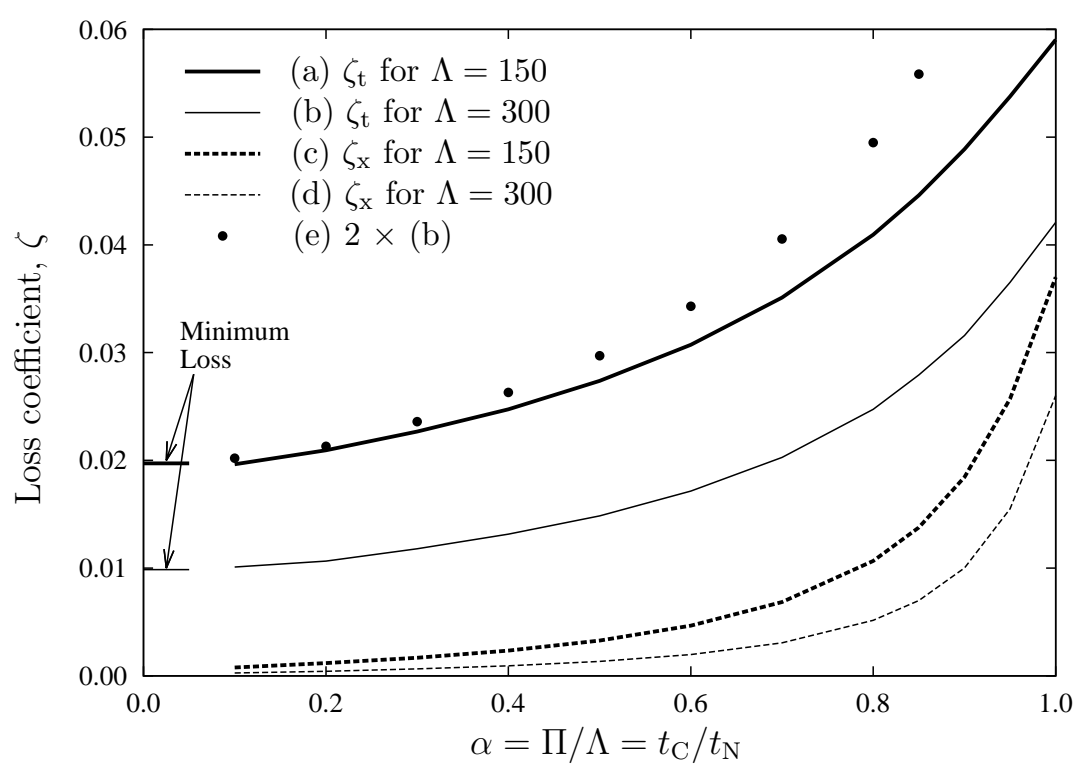

Figure 7. Computed loss coefficients for cyclic operation as a function of utilisation factor, $\alpha$, with $T_{1}=600 \mathrm{~K}$ and $T_{2}=T_{0}=300 \mathrm{~K}(\delta=2$ and $\phi=1)$. 


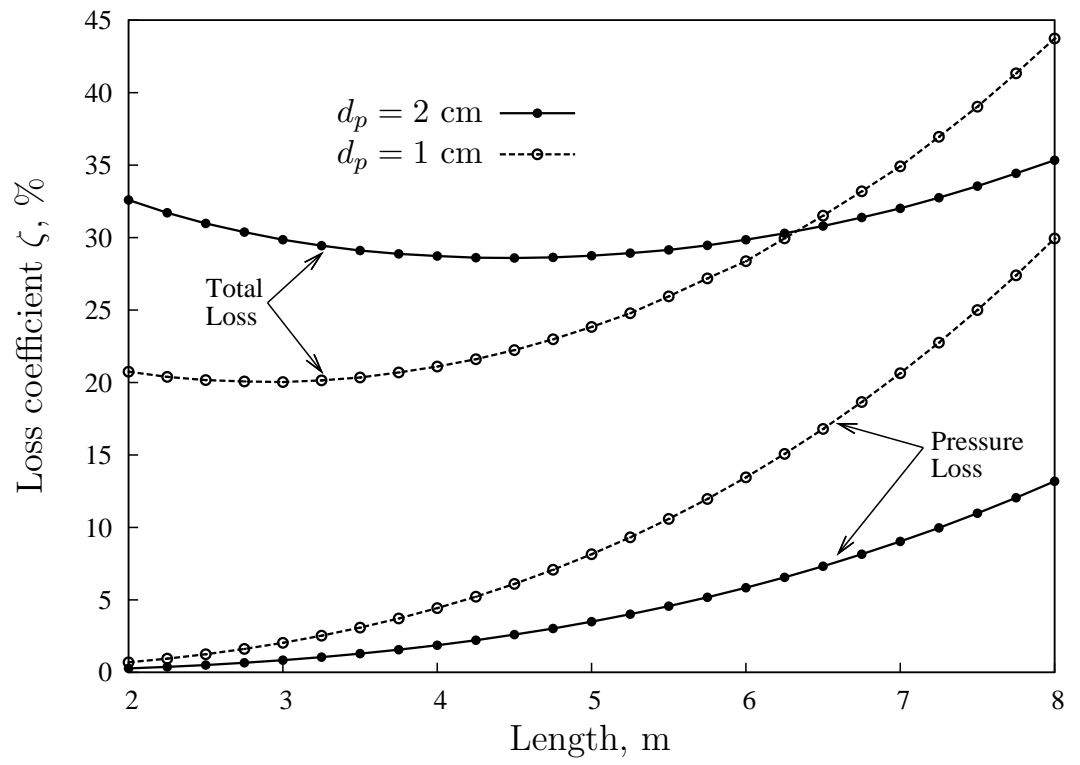

Figure 8. Variation of total loss with reservoir length at fixed volume and mass flow rate for a cold reservoir operated in single charge-discharge mode. Conditions are as specified in Table 2, but with the reservoir length and diameter allowed to vary whilst keeping volume constant. 


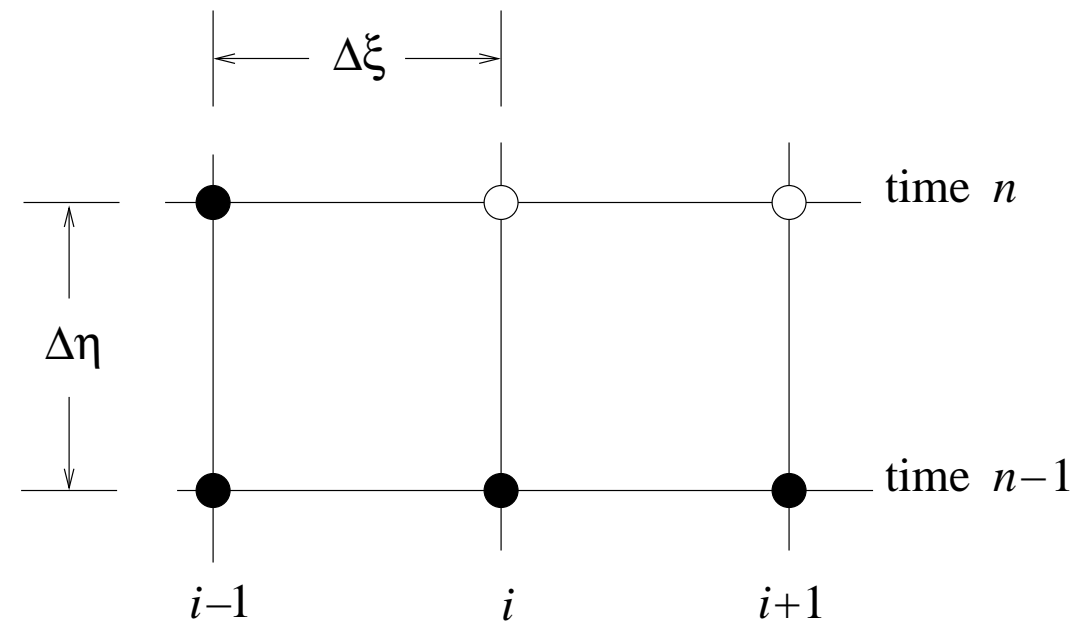

Figure 9. A section of the computational grid: temperatures are known at nodes marked $\bullet$ and unknown at nodes marked $\odot$. 